\title{
Thinking about idealization, abstraction and scientific models: an introduction
}

\section{Demetris Portides ${ }^{1}$}

Published online: 20 May 2021

(C) The Author(s), under exclusive licence to Springer Nature B.V. 2021

For a large part of the twentieth century the view that theories could be understood as rationally reconstructed formal axiomatic systems prevailed. According to this view the theoretical statements of the formal calculus stretched deductively all the way to observational statements. No representational role was assigned to scientific models. Their role was more or less cosmetic; they were identified with semantic models of the calculus that provided the interpretation of the logical syntax. The idealizations present in scientific theorizing were viewed as problems concerning the inferential connection between theoretical and observational statements. If a theory is conceived as a deductively closed set of statements and its axioms conceived as empirical universal generalizations, then to apply theory to phenomena, i.e. to deductively link theoretical to observational statements, the idealizations involved at the level of theory should somehow be accounted for; or, as Hempel (1988) put it: provisos are required in order to establish the deductive link. However, as Hempel argued, in many theory applications there would be an indefinitely large number of such provisos, thus trivializing the concept of scientific laws understood as empirical universal generalizations. In other cases, some provisos would not even be expressible in the language of the theory, thus making the deductive step impossible. Therefore, the argument that theory applications presuppose provisos undermines the view that theory relates to observation sentences deductively. The above view about scientific theories also faced several other well-known important problems (see, Suppe, 1974; Portides, 2017) that eventually led to its demise and opened the door for its successor, which is nowadays known as the Semantic or Model-theoretic view of scientific theories.

According to the Semantic View a scientific theory is identified with, or presented as, a class of models. An immediate consequence of identifying theory structure with classes of models is that models and modeling are turned into crucial

This set of papers is dedicated to the memory of Margaret Morrison (1954-2021), Professor of Philosophy at the University of Toronto (1989-2019).

Demetris Portides

portides@ucy.ac.cy

1 University of Cyprus, Nicosia, Cyprus 
components of scientific theorizing. Even though, as several authors have since argued (e.g. some of the contributors to the volume by Morgan \& Morrison, 1999), the thesis that models are an important component of scientific theorizing does not necessarily entail that theory structure can be identified with classes of models, this thesis were nevertheless a major contribution of the SV; for it put models and modeling to the forefront thus triggering reflection and debate on three interconnected philosophical issues: the nature and functions of scientific models, the character of scientific representation, and the bearing of the cognitive acts of idealization and abstraction on the functions of models and on the ways models represent phenomena. As a result, the last few decades, philosophers of science have been systematically analyzing and debating the concepts of model, representation and idealization/ abstraction.

These recent debates have shed much light on the idea that models are primary devices of scientific representation of target systems in the world, and that idealizations and abstractions are manifest in most (if not all) kinds of scientific representation. Scientific models are meant to represent something, whether physical or ideal systems or states. For example, an architectural model of a building is a representation of an actual (or to-be-actualized) building. Moreover, a scientific representation is not an exact copy of its target, but it omits many of the target's details and it simplifies others. Thus, 'model' is also linked to idealization and abstraction, i.e. a model represents a physical system in an abstract and idealized way. For example, a model of a building is not meant as an exact replica but as an idealized and abstract representation of an actual building (see for example Suppe, 1989) because, for instance, it represents only certain features of the actual system, e.g. the model of the building could represent the spatial relations and ignore other features such as the plumbing or electrical systems. Subsequently, it has become commonplace that scientific models, scientific representation and idealization/abstraction are entangled concepts.

However, disentangling the three concepts has proven to be anything but an easy task since these debates have also revealed, among other things, the difficulties in finding an all-inclusive and precise definition of the concepts of scientific model, scientific representation and idealization/abstraction. The route taken by most philosophers has, therefore, been to focus on some particular characteristics of this entanglement and attempt to explore these and their consequences in depth. This set of papers is no different in this respect, each author explores particular questions that pertain to this complex relation.

Arnon Levy, in his paper "Idealization and Abstraction: Refining the Distinction", gives a characterization of idealization and of abstraction in such a way so the two could be kept distinct; and, as he argues, these characterizations can be effective in clarifying various issues in Philosophy of science, such as scientific explanation. He defines abstraction as the measure, so to speak, of the level of detail present in a description, i.e. the more abstract a description the less detail it contains. Thus, rendering abstractions detail-poor representations. He defines idealization as deliberate misrepresentation of some aspect of the world, thus rendering idealizations as deliberate distortions. His distinction is a variant of Jones (2005) suggestion that idealization should be understood as being tied to falsehood and abstraction as being 
tied to truth. The main difference being that in Levy's distinction abstraction is not necessarily tied to truth. Levy uses his distinction in order to argue that abstraction should be kept distinct from generality.

Demetris Portides, in his paper "Idealization and Abstraction in Scientific Modeling", argues that the view advocated by Philosophers of Science, such as Jones (2005) and Godfrey-Smith (2009), that ties idealization to falsehood and abstraction to truth does not adequately characterize the distinction between the two. An important part of his argument relies on the idea that when a modeler abstracts away, she is not necessarily aware of the features that are omitted. He calls this cognitive act abstraction-as-extraction, and continues his argument showing that such a cognitive act cannot be tied to truth, and that it is best understood as a particular mode of selective attention applied on the set of features of the target. He further argues that idealization could be characterized as another mode of selective attention applied on the set of logically possible modifications of features of the target. He thus presents a distinction between the two based on the cognitive processes and not their conceptual products.

Steven French, in his paper "Identity Conditions, Idealisations and Isomorphisms: A Defence of the Semantic Approach", first dismisses the challenge to the Semantic Conception of scientific theories, by Halvorson (2012), that identity conditions for theories should be provided. He then moves on to address another challenge to the Semantic View, namely that the latter cannot capture the crucial feature of idealization, that is ubiquitous in scientific practices, and the various attributes of scientific representations that are consequences of idealizations. In particular, he defends the claim that the partial structures approach of the Semantic View does satisfy the set of criteria that Weisberg (2013) argues any account of scientific modeling must meet. Specifically, that any account of model-world relationship must distinguish between more successful and less successful representations, that the modelworld relationship should be construed as coming in degrees, that the model-world relationship should allow for qualitative as well as quantitative comparisons and it should be understood to depend on context.

Alisa Bokulich, in her paper "Using Models to Correct Data: Paleodiversity and the Fossil Record", takes up the issue of data models and gives an analysis of what takes place before theory or theoretical model confronts data, thus shedding much light on the complexities involved in constructing data models. She argues that it is the fidelity and not the purity of data that matters. By using the case of paleodiversity data models constructed from the fossil record, she shows that in order to achieve fidelity data must be corrected by removing artefactual elements and reducing noise. She also shows that all methods by which raw data are corrected involve the use of models. Thus, idealizing or, more broadly, simplifying assumptions are involved in correcting raw data. She makes use of this analysis to argue that corrected data can be more epistemically important than raw data (as is the case of paleodiversity data models), that fidelity of data is a matter of degree, that fidelity is judged relative to a particular purpose thus making it context-dependent, and that fidelity is often achieved by vicarious as opposed to physical control.

In their paper " Mathematics is not the only language in the book of nature", James Nguyen and Roman Frigg argue that the structuralist approach to the general 
application problem of mathematics to the world is wanting. What they call the general application problem is: "in virtue of what does a mathematical structure apply to a target system?" They first argue that the structuralist approach (with reference to the account of Bueno \& Colyvan, 2011) does not fully address this problem because in order to find a mapping between a mathematical structure and a target in the world, the target must exhibit a structure, as mappings exist between structures not between a structure and a physical object. Since a physical target does not exhibit a unique structure, they suggest that for the structuralist approach to work it must be supplemented with what they call the extensional abstraction account of generating a target structure. By this they mean that, following a physical description of the target and abstracting away to the extension of such a description, a target structure is generated that allows the application of mathematics to the world. Therefore, in order for the structuralist account to work as an explanation of how mathematics is applied to the world a structure generating description is necessary. Thus, as they put it, mathematics is not the only language in the book of nature.

Margaret Morrison, in her paper "Turbulence, Emergence and Multi-scale Modeling" discusses the phenomenon of emergence, explains that such phenomena are indeed characterized by non-linearity and complexity but argues that their most important ontological feature is universality. She also analyzes important aspects of multiscale modeling. In particular, she focusses on turbulence and on how it is modeled and shows why this phenomenon is not successfully captured by multi-scale modeling. Her argument is that the way turbulence is modeled shows that it does not exhibit the type of universality demonstrated by universality classes in Statistical Physics, thus it does not clarify how macro phenomena can emerge from and be independent from their microphysical base. This allows her to conclude that although the methodology of multi-scale modeling is particularly helpful in merging together models at different scales and possibly seeing how the latter relate to each other, it does not illuminate the micro/macro relation of emergent behavior.

William Bechtel, in his paper "Explaining Features of Fine-Grained Phenomena Using Abstract Analyses of Phenomena and Mechanisms: Two Examples from Chronobiology", argues that often a mechanism is either not needed or insufficient to provide the explanation required, thus rejecting the claim that mechanistic accounts alone explain. Often, he argues, the focus is in giving an explanation for a specific feature of fine-grained phenomena and not the general phenomenon itself. Such soughtafter explanations are not achieved by invoking models of mechanisms. Instead, the explanation is achieved either by abstractly representing the phenomenon for which an explanation is sought or by abstracting away from the details of a particular mechanism in order to explain why it produces the phenomenon. He argues for each of his conclusions by analyzing two respective examples from chronobiology.

\section{References}

Bueno, O., \& Colyvan, M. (2011). An inferential conception of the application of mathematics. Nous, 45(2), 345-374. 
Godfrey-Smith, P. (2009). Abstractions, idealizations and evolutionary biology. In A. Barberousse, M. Morange, \& T. Pradue (Eds.), Mapping the future of biology: Evolving concepts and theories (Boston Studies in the Philosophy of Science) (pp. 47-55). Springer.

Halvorson, H. (2012). What scientific theories could not be. Philosophy of Science, 79, 183-206.

Hempel, C. (1988). Provisos: A problem concerning the inferential function of scientific theories. In A. Grünbaum \& W. C. Salmon (Eds.), The limitations of deductivism (pp. 19-36). University of California Press.

Jones, M. (2005). Idealization and abstraction: A framework. In M. Jones \& N. Cartwright (Eds.), Idealization XII: Correcting the model, idealization and abstraction in the sciences (pp. 173-217). Rodopi.

Morgan, M. S., \& Morrison, M. (Eds.). (1999). Models as mediators: Perspectives on natural and social science. Cambridge University Press.

Portides, D. (2017). Models and theories. In L. Magnani \& T. Bartolotti (Eds.), Springer handbook for model-based science (pp. 25-48). Springer.

Suppe, F. (1974). The search for philosophic understanding of scientific theories. In F. Suppe (Ed.), 1977 The structure of scientific theories (pp. 1-241). University of Illinois Press.

Suppe, F. (1989). The semantic conception of theories and scientific realism. University of Illinois Press. Weisberg, M. (2013). Simulation and similarity. Oxford University Press.

Publisher's Note Springer Nature remains neutral with regard to jurisdictional claims in published maps and institutional affiliations. 